\title{
Identifying selective drug combinations using Comparative Network Reconstruction
}

\author{
Evert Bosdriesz ${ }^{1,2, *}$, João M. Fernandes Neto ${ }^{2}$, Anja Sieber ${ }^{3,4}$, René Bernards ${ }^{2}$, Nils \\ Blüthgen ${ }^{3,4,5}$, and Lodewyk F.A. Wessels ${ }^{1,6, *}$ \\ ${ }^{1}$ Computer Science Department, Center for Integrative Bioinformatics (IBIVU), Vrije Universiteit \\ Amsterdam, The Netherlands \\ ${ }^{2}$ Division of Molecular Carcinogenesis, The Oncode Institute, The Netherlands Cancer Institute, \\ Amsterdam, the Netherlands \\ ${ }^{3}$ Institute of Pathology, Charité Universitätsmedizin, Berlin, Germany \\ ${ }^{4}$ IRI Life Sciences, Humboldt University of Berlin, Berlin, Germany \\ ${ }^{5}$ Berlin Institute of Health, Berlin, Germany \\ ${ }^{6}$ Faculty of EEMCS, Delft University of Technology, Delft, the Netherlands \\ *Corresponding authors: e.bosdriesz@vu.nl, I.wessels@nki.nl
}

\begin{abstract}
Inhibition of aberrated signaling processes using targeting inhibitors is an important treatment strategy in cancer. However, due to network effects and the rapid onset of resistance, single drug treatments are often ineffective and responses short-lived. The use of multi-drug combinations has the potential to overcome these problems, but to avoid toxicity such combinations must be selective and the dosage of the individual drugs should be as low as possible while still being effective. Since the search space of possible multi-drug combinations is enormous, especially if the dosing needs to be optimized as well, a systematic method to identify the most promising combinations of drugs and dosages is required. We therefore developed a combined experimental and computational pipeline where we perform a limited set of drug-perturbation experiments, employ these to reconstruct mutantspecific signaling networks, and connect changes in signaling output to changes in cell viability. These models can then be used to prioritize selective low-dose multi-drug combinations in silico, based on the mutation profile of the target cell population. As a proof of principle, we applied this approach to a breast cell line and an isogenic clone with an activating PI3K mutation, for which we predicted and validated multiple selective multi-drug combinations. Applying this pipeline to suitably chosen models systems will allow for the identification of biomarker-specific combination treatment regimens.
\end{abstract}

\section{Introduction}

The dependency of tumors on activated signaling pathways results in therapeutic responses to inhibitors that block pathway activity [1]. However, resistance to such targeted inhibitors inevitably develops [2, 3]. Combinations of two targeted inhibitors can give more lasting clinical benefit, but resistance nonetheless emerges $[4,5]$. Combining more than two drugs might further extend the duration of the response [6], but toxicity becomes a major concern when multiple drugs are combined at their maximum tolerated dose (MTD). Recently, we found that partial inhibition of three or four kinases by combining Multiple drugs at Low Dose (MLD) is surprisingly effective in receptor tyrosine kinase (RTK) driven tumors in multiple cancer types. It prevents the development of resistance, and it is well tolerated by mice [7]. Others have also shown the potential of multi-drug (low dose) combinations in pre-clinical [8-11] and clinical $[12,13]$ settings.

These findings warrant further exploration of multiple-drug combination strategies and call for a systematic way to explore the opportunities, including optimizing the dosing of the different drugs. The 
combinatorial explosion of the search space - there are more than 2 million possible 4-way combinations of the 89 (as of 2017 [14]) FDA approved targeted inhibitors, and 24 billion if each drug is to be tested at 10 different concentrations - means that in-vitro testing of all combinations is infeasible and hence computational approaches are required to prioritize promising combinations. Nowak-Sliwinska and collaborators presented a "Feedback Systems Control" approach to explore the search-space of possible multi-drug combinations $[10,15,16]$. While this approach is promising, the method does not optimize for selectivity and the obtained results lack a mechanistic underpinning, making it hard to assess to what extent the results will generalize. Another promising approach is building mathematical models of cellular signaling, based on a limited set of (perturbation) experiments [17-24]. Unfortunately, current approaches suffer from two major shortcomings. First, only a very limited number of such modeling approaches focus on the difference between cells with different mutation profiles [17, 25], which is critical for optimizing selectivity. Second, how inhibition of oncogenic signaling affects proliferation and apoptosis quantitatively, remains underexplored $[23,24,26]$.

We therefore set out to establish and validate a combined experimental and computational pipeline to prioritize multi-drug low-dose combinations based on mathematical models of drug response (Fig 1). Importantly, we aimed to find combinations that are selective for cells with or without a particular mutation. To isolate the effect of the mutation, we used an isogenic cell line pair with and without a mutation. Specifically, we used MCF10A, a cell line derived from epithelial breast tissue [27], and an isogenic clone with the activating $\mathrm{PI} 3 \mathrm{~K}^{\mathrm{H} 1047 \mathrm{R}}$-mutation knocked in under its endogenous promoter [28]. We measured the response of the MAPK and AKT pathway and cell viability after drug perturbations, and used the measurement to build mutant specific signaling networks models using Comparative Network Reconstruction, a method we recently developed [17]. In addition, we parameterized the relation between signaling response and cell viability. Combining this allowed us to simulate the effect of arbitrary multidrug combinations and thus to prioritize promising ones. Our models indicated that no drug combination would likely be selective for the PI3K-mutant cells, but low-dose multi-drug combinations that we predicted to be selective for the parental cells were indeed selective, which validated our computational pipeline.

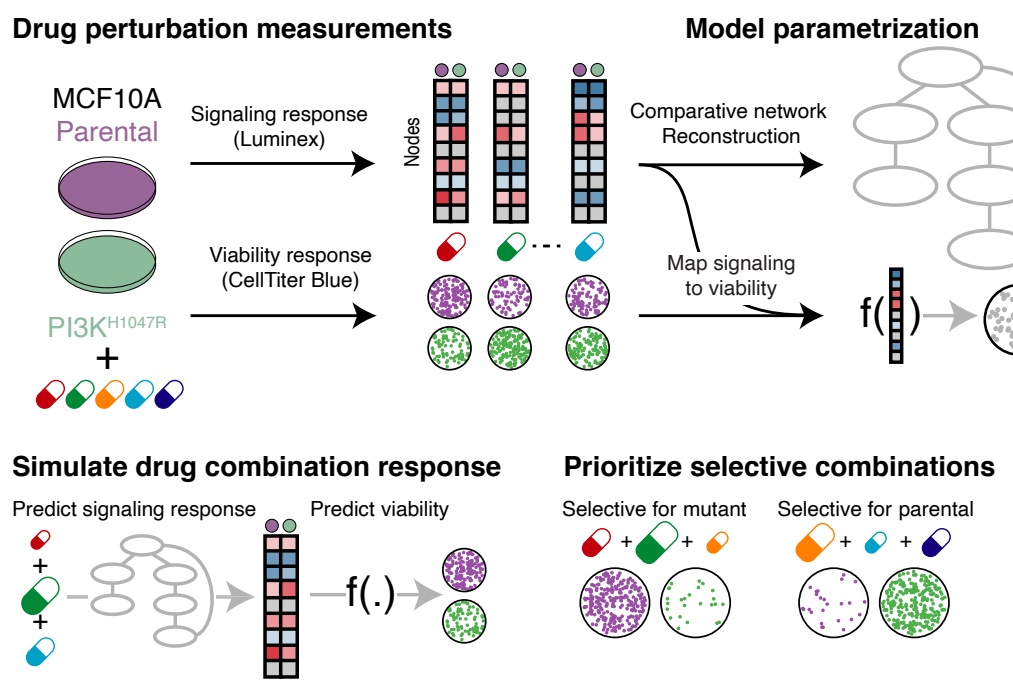

Figure 1: Overview of pipeline to prioritize promising selective low-dose multi-drug combinations. Top: MCF10A parental and PI3K ${ }^{\mathrm{H} 1047 \mathrm{R}}$ cells are treated with inhibitors targeting the MAPK and AKT pathways. The signaling and cell viability responses are measured and used to build mutant specific models of signal-transduction networks and to parametrize the relationship between signaling response and cell viability. Bottom: These models are used to simulate the response to unobserved multi-drug combinations, at arbitrary concentrations, of the signaling networks and how this affects cell viability. In this way, low-dose multi-drug combinations that are likely selective for a particular cell type can be prioritized. 


\section{Results}

\section{The signaling and viability response to drug perturbations in MCA10A parental and PI3K ${ }^{\mathrm{H} 1047 \mathrm{R}}$ mutated cells}

To test how oncogenic mutations affect signal transduction networks and their downstream effects on cellular phenotypes such as cell viability, we used the MCF10A cell line [27] and an isogenic clone with the activating $\mathrm{PI}_{3} \mathrm{~K}^{\mathrm{H} 1047 \mathrm{R}}$ mutation knocked in under its endogenous promoter [28]. In the absence of drug perturbations, $\mathrm{PI} 3 \mathrm{~K}^{\mathrm{H} 1047 \mathrm{R}}$-mutant MCF10A cells have a comparable growth rate as their parental cells [28]. As expected, the baseline signaling activity of AKT and PRAS40, both downstream of $\mathrm{PI} 3 \mathrm{~K}$, is elevated, but the other signaling nodes do not show significant differences in activity (Fig $2 \mathrm{~A}$ ). Dose response curves of selected PI3K and the MAPK pathway inhibitors showed subtle differences in sensitivities between the parental and the mutant cells (Fig S1A).

A Baseline signaling activity

B
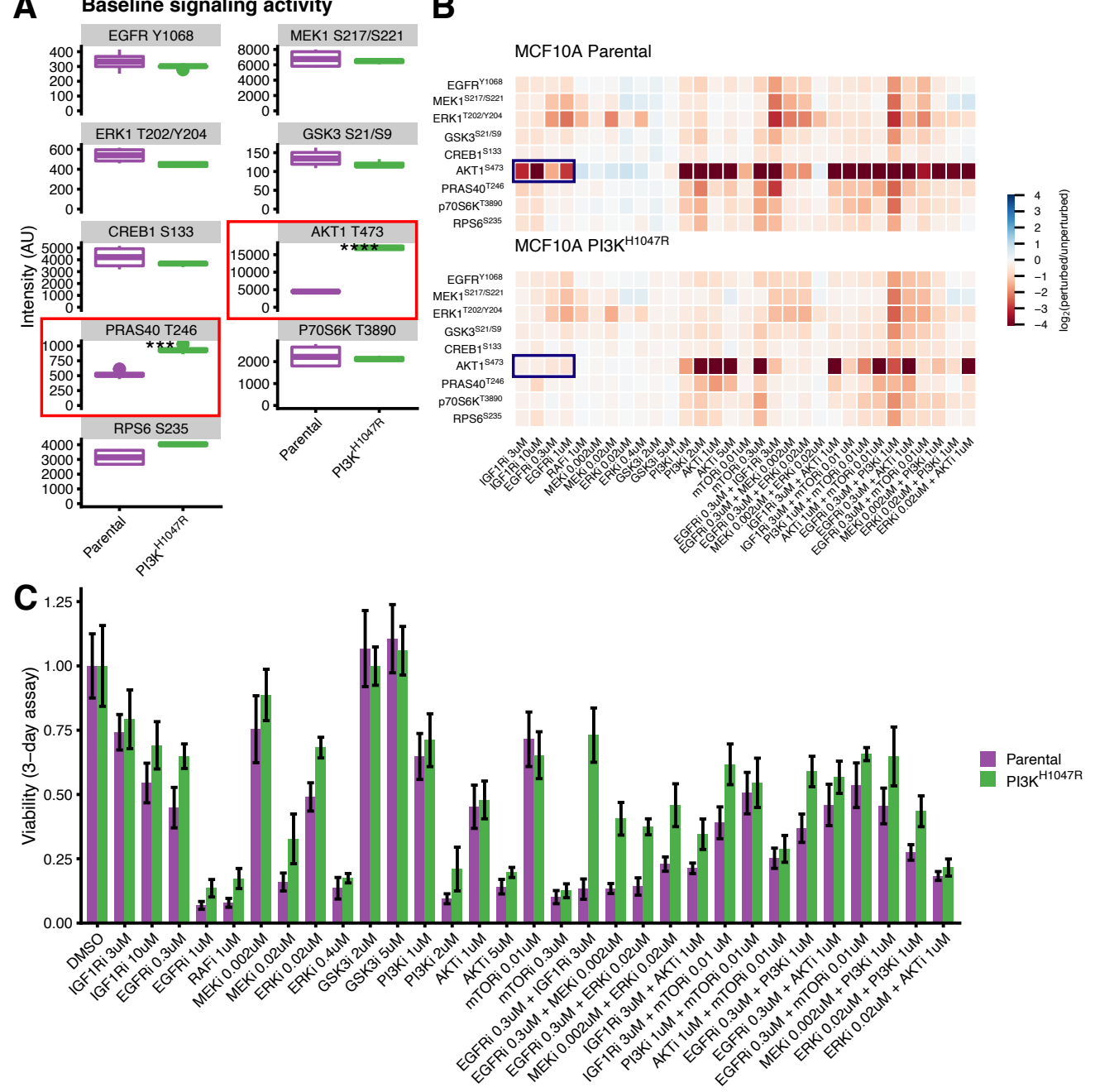

Figure 2: Profiling signaling and viability response of MCF10A Parental en $\mathrm{PI} 3 \mathrm{~K}^{\mathrm{H} 1047 \mathrm{R}}$ cells to drug perturbations. A. Node activity in the unperturbed cells. Most nodes have similar activity in the parental and PI3K $\mathrm{K}^{\mathrm{H} 1047 \mathrm{R}}$ cells, except AKT and PRAS40 (highlighted) which are downstream of PI3K. B. Heatmap representing $\log _{2}$-fold changes compared to DMSO controls of the signaling nodes in response to drug treatment. Signaling response is measured after 2 hours of drug treatment. The color scale is capped between -4 and 4 for visualization purposes. C. Cell viability under the same drug treatments as reported in panel B. Cell viability is measured after 3 days of drug treatment.

To study how the signaling of these cells respond to drug-perturbations and if the $\mathrm{PI} 3 \mathrm{~K}^{\mathrm{H} 1047 \mathrm{R}}$ mutation influences this, we perturbed both cell lines with inhibitors of the PI3K and MAPK pathways, and selected 
bioRxiv preprint doi: https://doi.org/10.1101/2020.12.17.423240; this version posted December 18, 2020. The copyright holder for this preprint (which was not certified by peer review) is the author/funder, who has granted bioRxiv a license to display the preprint in perpetuity. It is made available under aCC-BY-NC 4.0 International license.

2-drug combinations of these. Single drugs were tested at two different concentrations, corresponding roughly to their $\mathrm{IC}_{50}$ and $\mathrm{IC}_{90}$ values (except $\mathrm{RAFi}$, which was only tested at I $\mathrm{C}_{90}$ ) and drug-combinations were tested with both drugs at their $\mathrm{IC}_{50}$ values, to obtain a total of 34 different perturbations. We measured the response $\left(\log _{2}\right.$ fold change relative to DMSO control) of nine main nodes in the PI3K and MAPK signaling pathway using a multiplexed luminex assay in both cell lines to obtain more than 600 signaling drug-response measurements (Fig 2B, Table S1,S2). In addition we measured the effect on cell viability using CillTiter Blue (Fig 2C, Table S3,S4). Luminex quantification showed excellent concordance with Western Blots (Fig S1B). Generally, the differences in both signaling response and cell viability between the parental and PI3K-mutant cells were subtle but consistent. For instance, while the responses of the signaling nodes of the parental and $\mathrm{PI} 3 \mathrm{~K}^{\mathrm{H} 1047 \mathrm{R}}$ cells are strongly correlated (Fig $2 \mathrm{~B}$ and Fig S1C), phospho-AKT shows a strong negative response to growth-factor receptor inhibition (EGFRi or IGF1Ri) that is nearly absent in the PI3K ${ }^{\mathrm{H} 1047 \mathrm{R}}$ mutant cells (Fig 2B, highlighted).

\section{Network reconstructions identify relevant differences between parental and PI3K-mutant cells}

To establish how the PI3K ${ }^{\mathrm{H} 1047 \mathrm{R}}$ mutation affects the signal transduction network, we used the drugresponse measurements to perform Comparative Network Reconstruction (CNR) [17] of the MAPK and AKT pathways. CNR is a method we recently developed to reconstruct and quantify signaling networks and identify the most important quantitative differences between two or more cell lines. Prior knowledge about the network topology can be included, but the algorithm can also propose edges to be added to the network. The edge-weights are interpreted as the percent change in the downstream node activity in response to a $1 \%$ change in activity of the upstream node. Importantly, CNR identifies which edges are quantitatively different between the two cell lines.

We used the canonical MAPK and PI3K pathway interactions as prior information, and extended the network topology with 4 edges that were added in leave-one-out cross validation loop (Fig $3 \mathrm{~A}$ and Fig S2A). The model gave a good fit to the data (Pearson correlation $=0.91$ ) (Fig 3B). As expected, most of these differences are located close to AKT in the network (note, PI3K is not measured) (Fig 3A). Specifically, in the PI3K ${ }^{\mathrm{H} 1047 \mathrm{R}}$ cells, AKT is less sensitive to changes in EGFR and unresponsive to IGF1R inhibition (Fig 3A,C), which is consistent with PI3K being constitutively activated. Additionally, AKT is less responsive to PI3K and mTOR inhibition (Fig 3D,E). At the IC $\mathrm{I}_{50}$, AKT is also less sensitive to AKT inhibition, but when $\mathrm{AKTi}$ is applied at its $\mathrm{IC}_{90}$, the $\mathrm{PI} 3 \mathrm{~K}^{\mathrm{H} 1047 \mathrm{R}}$ cells show a larger response (Fig $3 \mathrm{~F}$ ). This last observation might be explained by the higher baseline AKT activity of PI3K ${ }^{\mathrm{H} 1047 \mathrm{R}}$ cells, since if AKT activity is reduced to a similar absolute level, the fold-change of AKT in the mutant is higher.

In order to be able to predict the signaling-response to drugs combined at arbitrary concentrations, we used the direct target-inhibition estimates for drug $k$ on node $i\left(s_{i k}\right)$ for the $\mathrm{IC}_{50}$ and $\mathrm{IC}_{90}$ to parameterized the general relation between target inhibition and drug concentration. To this end, we modeled the response as $s_{i k}\left(\left[I_{k}\right]\right)=I_{\max , i k} *\left[I_{k}\right] /\left(K_{I, i k}+\left[I_{k}\right]\right)$, where $\left[I_{k}\right]$ is concentration of inhibitor $k, I_{\max , i k}$ the maximal response of node $i$ to inhibitor $k$ (as $\left[I_{k}\right]$ goes to infinity) and $K_{I, i k}$ the inhibitor concentration where the half-maximal response is attained (Fig 3C-F and Fig S2B, dashed lines, c.f. Materials and Methods equation (4)).

\section{Short-term signaling response is informative for long-term cell viability}

To prioritize multi-drug combinations, the response of the signaling network needs to a drug perturbation needs to be related to its effect on cell viability. Important open questions here are: Is the short-term signaling response predictive to longer term cell viability? If so, which signaling outputs are most predictive?

The association between the individual node-responses and cell viability were moderate even for the most strongly associated nodes, phospho-AKT and phospho-ERK, which had a Pearson correlation with cell viability of 0.36 and 0.42 , respectively (Fig $4 \mathrm{~A}$ ). The responses of all other nodes also correlated somewhat with cell viability (Fig S3A), but clearly no single node alone is a good predictor for cell viability.

We therefore investigated whether a model combining the phospho-AKT and phospho-ERK response could fit the cell viability data better. We used a model with the property that cell viability goes to 0 if either phospho-ERK or phospho-AKT are fully inhibited (c.f. Materials and Methods, equation (3)). The 

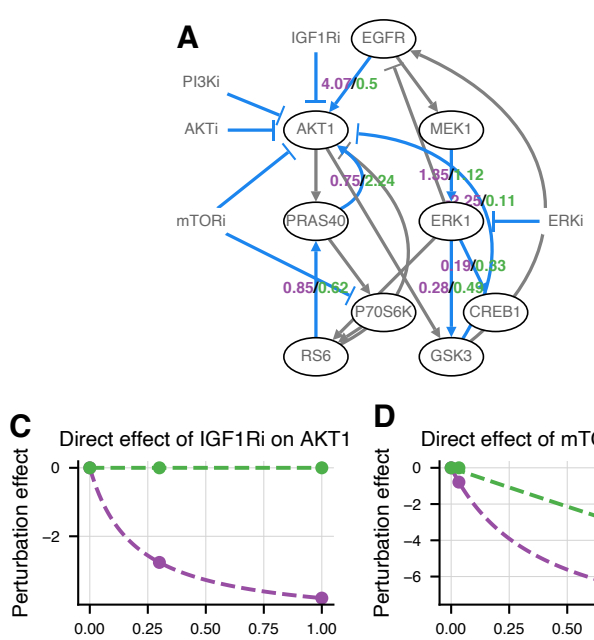

[IGF1Ri] (relative to IC90)
D

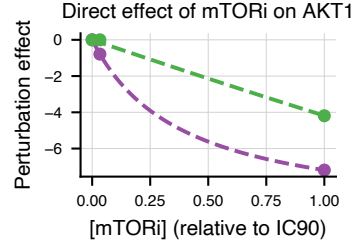

B
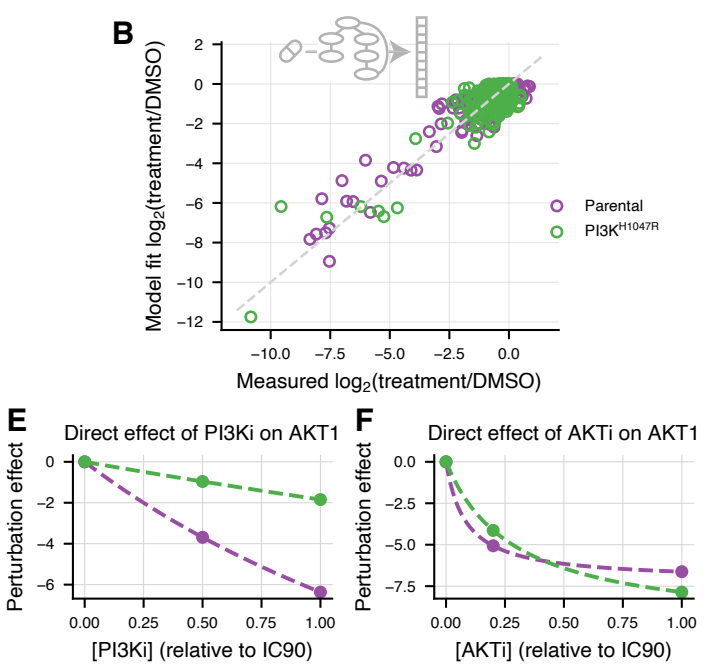

Figure 3: Mutant specific network reconstructions show expected differences. A. Comparative Network Reconstruction (CNR) of MCF10A parental and $\mathrm{PI} 3 \mathrm{~K}^{\mathrm{H} 1047 \mathrm{R}}$ cells. Edges and direct perturbation effects that differ between the two cell lines are highlighted in blue. Ovals indicate nodes. As expected, the most and the strongest differences between the cell lines are located close to AKT in the network (note that PI3K is not measured). B. Comparison of network model fit with measured signaling response shows that the network model can explain the signaling response data well (Pearson correlation 0.91). C-F. The estimated direct effect of IGF1R (C), mTOR (D), PI3K (E) and AKT (F) inhibition on AKT activity as a function of applied inhibitor concentration. Points indicate the estimated effects of the concentrations used in the CNR reconstruction, the dashed lines indicate the interpolated curves between these points (c.f. Materials and Methods, equation (4)). IGF1R PI3K, and mTOR inhibition were modelled as directly affecting AKT because their actual targets were not measured.

biological assumption behind this is that both ERK and AKT activation are required for cell survival and growth. This model gave a good fit to the data (Fig 4B), with a Pearson correlation between fitted and measured viability of 0.71 . Importantly, this means that the short-term signaling response is predictive for longer-term cell viability. The fitted parameters $\left(K_{M, E R K}\right.$ and $\left.K_{M, A K T}\right)$ differ slightly between the two cell lines, but the bootstrapped 95\% confidence intervals strongly overlap (Fig S3B), so we do not want to overinterpret these differences.
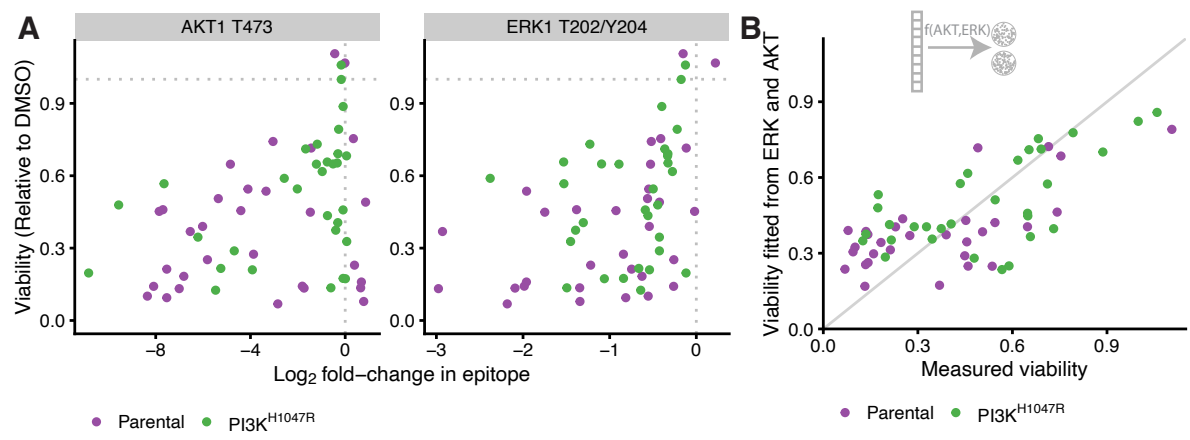

Figure 4: Short term signaling response is predictive for longer term cell viability. A. Scatterplot of cell viability against $\log _{2}$-fold changes in AKT (left panel) and ERK (right panel) activity in response to drug treatments. The Pearson correlations are 0.36 and 0.42 respectively. B. Scatterplot of model fit against measured cell viability based on a model where both ERK and AKT response are used to explain cell viability (c.f. Materials and Methods, equation (3)). The Peason correlation between fit and measurement is 0.71 .

\section{Prediction and validation of selective multi-drug-combinations}

We then combined the network models (Fig $3 \mathrm{~A}$ ) with the parametrization of the signaling-viability model (Fig $4 \mathrm{~A}$ ) to simulate the effect on cell viability of any multi-drug combination at any concentration. When 
applying this model to the training data, the Pearson correlation between measured and fitted cell viability was 0.78 (Fig $5 \mathrm{~A})$. We decided that this was accurate enough to make predictions about unseen multi-drug combinations. Hence we used our model to prioritize multi-drug combinations and dosings that maximize the selectivity, defined as the difference in viability between the two cell lines.
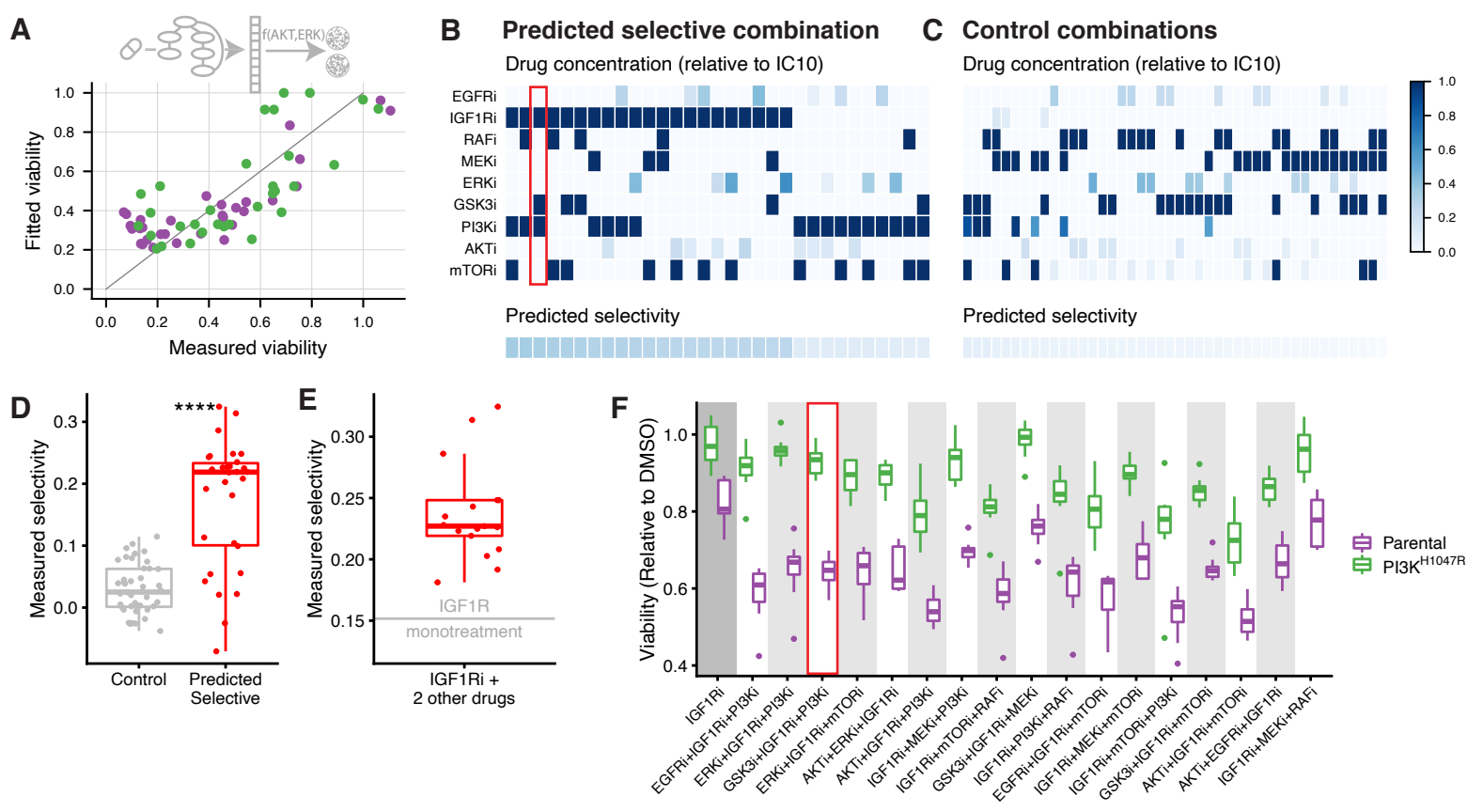

Figure 5: Experimental validation of selective drug combination predictions. A. Scatterplot comparing full model fit (network model combined with signaling response-viability mapping) to the training data. The Peason correlation between fit and measurement is 0.78 . B-C. Overview of drug combinations that we predicted to be selective $(B)$ and non-selective $(C)$ based on this model. Drug concentrations are color-coded relative to their $I C_{10}$. Bottom row indicates predicted selectivity (defined as the difference in viability between $\mathrm{PI} 3 \mathrm{~K}^{\mathrm{H} 1047 \mathrm{R}}$ and parental cells) of the combination. These combinations were subsequently tested in the validation experiments. D. Boxplot comparing the measured selectivity of the drug combinations predicted to selective (panel B) or non-selective (panel $\mathrm{C}$ ). Each point represents the mean selectivity of one drug combination, which were each tested in 8 replicates. The difference is highly significant (Wilcoxon signed-rank test $p<10^{-7}$ ). E. Comparison of the measured selectivity of IGFRi mono treatment, indicated by the horizontal gray line, with the selected IGF1Ri-containing 3-drug combinations (red boxplot). IGF1Ri containing combinations are significantly more selective than IGF1Ri mono treatment (onesample t-test $p<10^{-7}$ ). F. Box plots comparing cell-viability of Parental and PI3K ${ }^{\mathrm{H} 1047 \mathrm{R}}$ cells of the 11 (out of 17) IGF1Ri containing drug-combinations that are significantly more selective than IGF1Ri mono treatment.

We first tried to optimize for PI3K ${ }^{\mathrm{H} 1047 \mathrm{R}}$-selectivity. To do this, for all possible 3-drug combinations we optimized the concentrations such that the viability of the PI3K ${ }^{\mathrm{H} 1047 \mathrm{R}}$ mutants is minimized, under the constraint that the viability of the parental cells remains above 0.8 relative to DMSO control (c.f. Materials and Methods, equation (6)). To look for low-dose drug combinations, we also added the constraint that each drug can be used maximally at its $\mathrm{IC}_{10}$. However, no drug-combination was predicted to be selective for the PI3K $\mathrm{K}^{\mathrm{H} 1047 \mathrm{R}}$ cells at any combination of concentrations. Since none of the single drugs shows selectivity towards the $\mathrm{PI} 3 \mathrm{~K}^{\mathrm{H} 1047 \mathrm{R}}$ cells (Fig 2C), this is not very surprising. Moreover, our network reconstructions indicated that the main effect of the $\mathrm{PI} 3 \mathrm{~K}^{\mathrm{H} 1047 \mathrm{R}}$ mutation is to render the MCF10A parental line independent of growth-factor stimulation. Indeed, when we grew MCF10A parental and $\mathrm{PI} 3 \mathrm{~K}^{\mathrm{H} 1047 \mathrm{R}}$ cells in the media without growth-factor, this is what we observed (Fig 4).

We then looked for drug-combinations that we predicted to be selective for the parental cells. In our optimizations, we found 30 such combinations with a selectivity $>0.1$ (Fig 4B). Interestingly, IGF1R inhibition was part of all of the 17 combinations that we predict to be most selective, while its selectivity in the training data was only modest (Fig $2 \mathrm{C}$ ). However, the difference in signaling response, and specifically phospho-AKT, was much more pronounced (Fig 2B), and this latter aspect gets picked up in the network reconstructions (Fig 3A). A particularly interesting example is the combination IGF1Ri + PI3Ki + GSK3i, since both PI3Ki and GSK3i at their lower dose $\left(\mathrm{IC}_{50}\right)$ show no selectivity towards the parental cells, 
bioRxiv preprint doi: https://doi.org/10.1101/2020.12.17.423240; this version posted December 18, 2020. The copyright holder for this preprint (which was not certified by peer review) is the author/funder, who has granted bioRxiv a license to display the preprint in perpetuity. It is made available under aCC-BY-NC 4.0 International license.

and yet this combination is predicted to be one of the more selective ones (Fig $5 \mathrm{~B}$, highlighted). As a control, we also selected 44 combinations that we predicted to be non-selective for either cell line (Fig 5C). We then treated the parental and PI3K $\mathrm{K}^{\mathrm{H} 1047 \mathrm{R}}$ cells with the 30 predicted to be selective and 44 control combinations and measured their viability (Table S5). Combinations that we predicted to be selective were indeed so, and this was highly significant when compared to the non-selective control combinations (Wilcoxon signed-rank test $p<10^{-7}$ ) (Fig 5D). Individually, 25 of the 30 combinations predicted to be selective were indeed significantly selective (one-sided t-test $p<0.05$ ) (Table S6).

As mentioned above, of the 30 predicted-to-be-selective combinations we tested, 17 contain the IGF1R inhibitor, which is also mildly selective for parental cells as monotherapy. (None of the other inhibitors showed selectivity as a monotherapy at their $\mathrm{IC}_{10}$, Fig S5). To rule out the possibility that our result is mainly driven by the selectivity of IGF1R mono-therapy, we compared the 17 IGF1Ri containing drug combinations with IGF1Ri monotherapy. Figure 5E shows that each of the IGF1Ri containing combinations we tested (red boxplot) is more selective than IGF1Ri treatment alone (indicated by horizontal gray line). This effect is highly significant (one-sample t-test $p<10^{-7}$ ). When looking at the individual drug combinations, we found that 11 of the 17 IGF1Ri containing combination treatments are significantly more selective than IGF1Ri monotherapy (one sided t-test $p<0.05$ ) (Fig 5E, Table S7). This also includes the IGF1Ri + PI3Ki + GSK3i combination highlighted above, which is amongst second most selective combination when ranked by effect size.

These results indicate that our pipeline is capable of making an accurate prioritization of selective low-dose multi-drug combinations. Importantly, these predictions are not always obvious, and would not have been possible without the help of mathematical models of the signal transduction networks and its relation to cell viability.

\section{Discussion}

In this study, we have shown that it is possible to prioritize promising multiple low dose (MLD) drug combinations based on a combination of single and 2-drug response measurements and mathematical modeling. We have used drug-perturbation experiments to reconstruct, quantify and compare signaltransduction networks of an isogenic cell line pair, and linked the responses of these networks to cell viability. This showed that the short-term signaling response is predictive for cell viability, which is measured in longer-term experiments. Based on the so-obtained models we were able to predict and validate drug combinations that are selective for a particular cell line, even though the differences with between the cell lines were subtle.

According to our model, no drug combination is likely to be selective for the $\mathrm{PI} \mathrm{K}^{\mathrm{H} 1047 \mathrm{R}}$-mutant MCF10A cells compared to their parental counterparts. The absence of oncogene-specific sensitivities is presumably due to an absence of "oncogene addiction" [1] to the PI3K mutation (or any other) in the PI3K ${ }^{\mathrm{H} 1047 \mathrm{R}}$ MCF10A cells. In the absence of drug-treatment the mutation has no effect on proliferation (under the growth conditions we used), and this mutation therefore presumably does not induce any vulnerabilities. Our network reconstruction suggests that the main effect of the $\mathrm{PI} 3 \mathrm{~K}^{\mathrm{H} 1047 \mathrm{R}}$ mutation on MCF10A cells is to make them growth-factor independent, consistent with previous observations [29] and likely the reason that IGF1R inhibition selectively affects the parental cells. Interestingly, while as monotherapy low-dose IGF1Ri is mildly selective, combinations of IGF1Ri with selected other drugs are strongly selective for the parental cells. Importantly, which combinations are most selective is often far from obvious. For instance, while in the training data PI3Ki and GSK3i at their lower dose $\left(\mathrm{IC}_{50}\right)$ individually show no selectivity towards the parental cells at all, the combination IGF1Ri + PI3Ki + GSK3i is one of the most selective drug combinations, both as predicted by our model and as measured validation experiments. This underscores the need for mathematical modelling in prioritizing promising combinations.

While isogenic cell line pairs with a mutation knocked in are interesting models because they allow study of the effect of the mutation in isolation, they may not always be the best model to study oncogenespecific sensitivities due to their lack of oncogene addiction. An interesting alternative approach might be to use cancer cell lines of which one of the driver mutations is removed [30-32]. Alternatively, a larger, more heterogeneous panel of cell lines with and without a particular biomarker could be used [19, 22, $33,34]$. In this scenario, one would look at commonalities in the signaling network response of the cell 
bioRxiv preprint doi: https://doi.org/10.1101/2020.12.17.423240; this version posted December 18, 2020. The copyright holder for this preprint (which was not certified by peer review) is the author/funder, who has granted bioRxiv a license to display the preprint in perpetuity. It is made available under aCC-BY-NC 4.0 International license.

lines with the biomarker compared to the lines without out, and use this to propose combinations that are selective of the biomarker carrying cell lines. Finally, the use of matched tumor and normal organoids from the same patient could be used for truly personalized models.

In conclusion, we have here shown that it is feasible to make accurate, non-trivial predictions about selectivity of multi-drug combinations based on mathematical models of signaling transduction networks. In combination with suitable model systems, this framework therefore makes it possible to rationally design biomarker-selective low-dose multidrug combinations.

\section{Materials and Methods}

\section{Cells and cell culture}

Human parental and PI3K $\mathrm{K}^{\mathrm{H} 1047 \mathrm{R}} /+\mathrm{MCF} 10 \mathrm{~A}$ cell lines were obtained from Horizon discovery (HD PAR003 and HD 101-011). Cells were cultured in DMEM/F-12 including $2.5 \mathrm{mM} \mathrm{L-glutamine} \mathrm{and} 15 \mathrm{mM}$ HEPES, supplemented with $5 \%$ horse serum, $10 \mu \mathrm{g} / \mathrm{mL}$ insulin, $0.5 \mu \mathrm{g} / \mathrm{mL}$ hydrocortisone and $0.1 \mu \mathrm{g} / \mathrm{mL}$ cholera toxin. Mycoplasma tests were performed every 2 months.

\section{Reagents and compounds}

The following inhibitors were used in this study: EGFRi (Gefitinib), IGF1Ri (OSI-906), RAFi (LY3009120), MEKi (Trametinib), ERKi (SCH772984), PI3Ki (BKM120), AKTi (MK-2206), mTORi (AZD8055). All inhibitors were purchased from MedKoo Biosciences. The luminex antibodies against CREB1 ${ }^{\mathrm{S} 133}$,

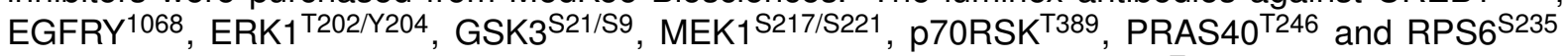
were purchased from ProtATonce $L t d$. The luminex antibody against $A K T 1^{\top 473}$ was purchased from BioRad.

\section{Drug perturbation and validation experiments}

All the cell-viability measurements were performed in biological triplicates, each with 2 technical replicates, using black-walled 384-well plates (Greiner 781091). Cells were plated at the optimal seeding density (200 cells per well) and incubated for approximately 24 hours to allow attachment to the plate. Drugs were then added to the plates using the Tecan D300e digital dispenser. $10 \mu \mathrm{M}$ phenylarsine oxide was used as positive control ( $0 \%$ cell viability) and DMSO was used as negative control ( $100 \%$ cell viability). Three days later, culture medium was removed and CellTiter-Blue (Promega G8081) was added to the plates. After 2 hours incubation, measurements were performed according to manufacturer's instructions using the EnVision (Perkin Elmer). Viabilities were normalized per cell line according to (treatment $\left.\mathrm{PAO}_{\text {mean }}\right) /\left(\mathrm{DMSO}\right.$ mean $\left.-\mathrm{PAO}_{\text {mean }}\right)$. $\mathrm{IC}_{50}$ and IC 90 values were fitted using the R-package MixedIC50 [35] (code available at https://github.com/NKI-CCB/MixedIC50).

The signaling response measurements were performed using 6-well plates (Greiner 657165). 300K cells per well were plated and incubated for approximately 24 hours to allow attachment to the plate. Drugs were then added to the plates and protein was harvested after 2 hours using the Bio-Plex Pro Cell Signaling Reagent Kit (BioRad 171304006M) according to the manufacturer's instructions. Protein concentration of the samples was normalised after performing a Bicinchoninic Acid (BCA) assay (Pierce BCA, Thermo Scientific), according to the manufacturer's instructions. Cell lysates were analyzed using the Bio-Plex Protein Array system (Bio-Rad, Hercules, CA) according to the suppliers protocol as described previously [19]. Intensities were normalized by subtracting blanks for each epitope and correcting for protein concentration.

\section{Computational pipeline and data analysis}

\section{Comparative network reconstruction}

MAPK and AKT signaling networks of the parental and PI3K ${ }^{\mathrm{H} 1047 \mathrm{R}}$ mutant cell lines were reconstructed based on the Luminex drug-response data using Comparative Network Reconstruction (CNR)[17]. Briefly, CNR is a network reconstruction method based on Modular Response Analysis [36]. It links the matrix 
of measured node responses to a set of perturbations, $\mathbf{R}$ (where $R_{i k}$ is defined as $\log _{2}$ fold change in node $i$ in response to perturbation $k$ ) to the matrix unobserved interaction strengths $\mathbf{r}$ (where $r_{i j}$ is the logarithmic partial derivative of node $i$ with respect to node $j$ ) and direct perturbation effects $\mathbf{s}$ (with $s_{i k}$ the scaled direct effect of perturbation $k$ on node $i$ ). These matrices are related through

$$
\mathbf{r} \cdot \mathbf{R}=-\mathbf{s}
$$

CNR solves this equation using an optimization procedure with penalties on the number of edges (non-zero entries in $\mathbf{r}$ ) and differences between cell lines (entries in $\mathbf{r}$ that are quantitatively different between the cell lines). The optimization problem reads:

$$
\begin{aligned}
& \text { Minimize: } \quad \sum_{n} \sum_{i, j} \sum_{x} \epsilon_{i n}^{\times 2}+\eta \cdot I_{i j}^{\text {edge }}+\theta \cdot\left(I_{i j}^{\text {diff }}+I_{i n}^{\text {sdiff }}\right) \\
& \text { Subject to: } \sum_{k} r_{i k}^{x} \cdot R_{k n}^{x}+s_{i n}^{x}=\epsilon_{i n}^{x} \\
& I_{i j}^{\text {edge }}=0 \Rightarrow r_{i j}^{\times}=0 \\
& I_{i j}^{\text {diff }}=0 \Rightarrow r_{i j}^{x}-r_{i j}^{\text {mean }}=0 \\
& I_{i n}^{\text {sdiff }}=0 \Rightarrow s_{i n}^{\times}-s_{i n}^{\text {mean }}=0 \\
& r_{i j}^{\text {mean }}=1 / N_{\text {cell lines }} \sum_{x} r_{i j}^{x} \\
& s_{i n}^{\text {mean }}=1 / N_{\text {cell lines }} \sum_{x} s_{i n}^{x} \\
& I^{\text {edge }}, I^{\text {dif }}, I^{\text {sdiff }} \in\{0,1\} \\
& \left.n \in\{\text { perturbations }\} ; i, j, k \in\{\text { nodes }\} ; \in \text { parental, } \mathrm{PI} 3 \mathrm{~K}^{\mathrm{H} 1047 \mathrm{R}}\right\}
\end{aligned}
$$

Solving this optimization problem gives the matrices $\mathbf{r}$ and $\mathbf{s}$ from a given $\mathbf{R}$.

Additional constraints reflecting the experimental design were added to the CNR problem.

- $s_{i k}$ is negative and stronger for higher drug concentrations, i.e. $0>s_{i k}\left(\left[I C_{50}\right]\right)>s_{i k}\left(\left[I C_{90}\right]\right)$.

- Each inhibitor-target pair has a single indicator for both inhibitor concentrations for the difference in perturbations strengths, i.e. if $I_{i k}^{\text {sdiff }}=0 \Rightarrow s_{i k}^{\text {parental }}\left(\left[I C_{50}\right]\right)=s_{i k}^{\text {PlsK }}\left(\left[I C_{50}\right]\right)$ and $s_{i k}^{\text {parental }}\left(\left[I C_{90}\right]\right)=$ $s_{i k}^{\text {Pl3K }}\left(\left[I C_{90}\right]\right)$.

- The MEK inhibitor interferes not only with MEK phosphorylation, but also its catalytic efficiency. Hence, MEK inhibition was modelled as a direct effect on both MEK and it's downstream proteins [17].

- Some inhibitors target kinases that were not measured in our assay. The effect of these inhibitors was modelled as a perturbation to the (canonical) downstream nodes of the kinases being inhibited. Specifically, IGF1R inhibition was modelled as a perturbation to MEK1 and AKT1, PI3K inhibition as a perturbation to AKT1, RAF inhibition as a perturbation to MEK1, and mTOR inhibition as a perturbation to AKT1 and p70S6K.

Prior information about network topology was added by setting the indicators of a set of canonical MAPK and PI3K pathway interactions to 1 . Hyperparameter were set to $\eta=0.1$ and $\theta=2.0$ based on a leave one out cross validation loop. Single drug treatments were not included in the leave one out cross validation because each drug concentration needs to be present in at least one perturbation to estimate the corresponding parameter. The final model was obtained by restricting the topology to the prior network information with addition of the 4 edges that were identified in the leave one out cross-validation, and then performing the optimization with $\theta=2.0$.

\section{The relation between signaling output and cell viability}

The viability (relative to DMSO control) upon perturbation $k, v_{k}$, were fitted to the following function:

$$
v_{k}=\frac{1}{1-R_{A K T, k} / K_{M, A K T}-R_{E R K, k} / K_{M, E r k}}
$$


Where $R_{A K T, k}$ and $R_{E R K, K}$ are the $\log _{2}$-fold changes of phospho-AKT and phospho-ERK relative to DMSO control upon perturbation $k$, respectively. Fitting was performed using the nls function of $R$. Bootstraps were performed using the function bootstrap from the "rsample" package [37].

\section{Simulation and prediction of selective 3-drug combinations}

The relations between the applied concentration of drug $k,\left[I_{k}\right]$, and target inhibition of node $i$ in response to this, $s_{i k}$ were fitted to the following function for each inhibitor-target pair,

$$
s_{i k}\left(\left[I_{k}\right]\right)=\frac{I_{\max , i k} *\left[I_{k}\right]}{\left(K_{I, i k}+\left[I_{k}\right]\right)} .
$$

The parameters $I_{\max , i k}$ and $K_{I, i k}$ were fitted to this function using the $s_{i k}$-values for the $\left[I_{k}\right]=I C_{50}$ and $I C_{90}$ obtained from the CNR optimizations with the curve_fit function from the python package "scipy.optimize" package [38]. For convenience all drug concentrations were normalized to the highest concentration applied (the $I C_{90}$ ), and in all analyses only interpolations and not extrapolations are used $(0 \leq[I] \leq 1)$.

$\mathbf{R}_{A+B+C}$, the vector of simulated $\log _{2}$-fold changes in response to a perturbation with 3 drugs $A, B$ and $C$, at concentration $\left[I_{A}\right],\left[I_{B}\right]$ and $\left[I_{C}\right]$ was calculated as

$$
\mathbf{R}_{A+B+C}=\mathbf{r}^{-1}\left(\mathbf{s}_{A}\left(\left[I_{A}\right]\right)+\mathbf{s}_{B}\left(\left[I_{B}\right]\right)+\mathbf{s}_{C}\left(\left[I_{C}\right]\right)\right),
$$

to obtain $R_{A K T, A+B+C}$ and $R_{E R K, A+B+C}$. These were then used to calculate viability according to equation (3).

For each possible 3-drug combination, the selectivity for cell line $x$ relative to $y$ was optimized by solving the following optimization problem:

$$
\begin{aligned}
\text { Minimize: } & v_{A+B+C}^{x} \\
\text { Subject to: } & v_{A+B+C}^{y} \geq 0.8 \\
& 0<\left[I_{k}\right]<I C_{10} \quad k \in\{A, B, C\}
\end{aligned}
$$

Similarly, unselective control combinations were obtained by solving the optimization problem:

$$
\begin{aligned}
\text { Minimize: } & \left(v_{A+B+C}^{\times}-0.8\right)^{2}+\left(v_{A+B+C}^{y}-0.8\right)^{2} \\
\text { Subject to: } & 0<\left[I_{k}\right]<I C_{10} \quad k \in\{A, B, C\}
\end{aligned}
$$

for all possible 3-drug combinations.

The optimizations were performed in Wolfram Mathematica [Mathematica] (version 12.0) using the NMinimize function.

\section{Data and Code availability}

All data and code required to reproduce the results and figures in this paper are available at https://github.com/evertbosdriesz/cnr-selective-combos.

\section{Ackowledgements}

This work was supported by funding from the Oncode Institute and the Gravity Program CGC.nl, funded by The Netherlands Organisation for Scientific Research (NWO).

\section{References}

1. Weinstein, I. B. Addiction to Oncogenes-the Achilles Heal of Cancer. en. Science 297, 63-64 (July 2002). 
bioRxiv preprint doi: https://doi.org/10.1101/2020.12.17.423240; this version posted December 18, 2020. The copyright holder for this preprint (which was not certified by peer review) is the author/funder, who has granted bioRxiv a license to display the preprint in perpetuity. It is made available under aCC-BY-NC 4.0 International license.

2. Holohan, C., Van Schaeybroeck, S., Longley, D. B. \& Johnston, P. G. Cancer Drug Resistance: An Evolving Paradigm. en. Nature Reviews Cancer 13, 714-726 (Oct. 2013).

3. Konieczkowski, D. J., Johannessen, C. M. \& Garraway, L. A. A Convergence-Based Framework for Cancer Drug Resistance. Cancer Cell 33, 801-815 (May 2018).

4. Flaherty, K. T. et al. Improved Survival with MEK Inhibition in BRAF-Mutated Melanoma. New England Journal of Medicine 367, 107-114 (July 2012).

5. Long, G. V. et al. Combined BRAF and MEK Inhibition versus BRAF Inhibition Alone in Melanoma. New England Journal of Medicine 371, 1877-1888 (Nov. 2014).

6. Boshuizen, J. \& Peeper, D. S. Rational Cancer Treatment Combinations: An Urgent Clinical Need. en. Molecular Cell 78, 1002-1018 (June 2020).

7. Fernandes Neto, J. M. et al. Multiple Low Dose Therapy as an Effective Strategy to Treat EGFR Inhibitor-Resistant NSCLC Tumours. en. Nature Communications 11, 3157 (June 2020).

8. Ryan, M. B. et al. Vertical Pathway Inhibition Overcomes Adaptive Feedback Resistance to KRASG12C Inhibition. en. Clinical Cancer Research, clincanres.3523.2019 (Nov. 2019).

9. Ozkan-Dagliyan, I. et al. Low-Dose Vertical Inhibition of the RAF-MEK-ERK Cascade Causes Apoptotic Death of KRAS Mutant Cancers. en. Cell Reports 31, 107764 (June 2020).

10. Zoetemelk, M. et al. Optimized Low-Dose Combinatorial Drug Treatment Boosts Selectivity and Efficacy of Colorectal Carcinoma Treatment. en. Molecular Oncology n/a (2020).

11. Caumanns, J. J. et al. Low-Dose Triple Drug Combination Targeting the PI3K/AKT/mTOR Pathway and the MAPK Pathway Is an Effective Approach in Ovarian Clear Cell Carcinoma. en. Cancer Letters 461, 102-111 (Oct. 2019).

12. Van Cutsem, E. et al. BEACON CRC Study Safety Lead-in (SLI) in Patients with BRAFV600E Metastatic Colorectal Cancer ( $\mathrm{mCRC}$ ): Efficacy and Tumor Markers. Journal of Clinical Oncology 36, 627-627 (Feb. 2018).

13. Van Cutsem, E. et al. Binimetinib, Encorafenib, and Cetuximab Triplet Therapy for Patients With BRAF V600E-Mutant Metastatic Colorectal Cancer: Safety Lead-In Results From the Phase III BEACON Colorectal Cancer Study. en. Journal of Clinical Oncology 37, 1460-1469 (June 2019).

14. Sun, J. et al. A Systematic Analysis of FDA-Approved Anticancer Drugs. BMC Systems Biology 11, 87 (Oct. 2017).

15. Nowak-sliwinska, P. et al. Optimization of Drug Combinations Using Feedback System Control. Nature Protocols 11, 302-315 (2016).

16. Weiss, A. et al. A Streamlined Search Technology for Identification of Synergistic Drug Combinations. Scientific reports 5, 14508 (2015).

17. Bosdriesz, E. et al. Comparative Network Reconstruction Using Mixed Integer Programming. en. Bioinformatics 34, i997-i1004 (Sept. 2018).

18. Dorel, M. et al. Modelling Signalling Networks from Perturbation Data. en. Bioinformatics (2018).

19. Klinger, B. et al. Network Quantification of EGFR Signaling Unveils Potential for Targeted Combination Therapy. Molecular systems biology 9 (Jan. 2013).

20. Halasz, M., Kholodenko, B. N., Kolch, W. \& Santra, T. Integrating Network Reconstruction with Mechanistic Modelling to Predict Cancer Therapy. Science signaling 9, ra114 (2016).

21. Saez-Rodriguez, J. et al. Discrete Logic Modelling as a Means to Link Protein Signalling Networks with Functional Analysis of Mammalian Signal Transduction. Molecular systems biology 5, 331 (Jan. 2009).

22. Jastrzebski, K. et al. Integrative Modeling Identifies Key Determinants of Inhibitor Sensitivity in Breast Cancer Cell Lines. en. Cancer Research 78, 4396-4410 (Aug. 2018).

23. Kirouac, D. C. et al. Computational Modeling of ERBB2-Amplified Breast Cancer Identifies Combined ErbB2/3 Blockade as Superior to the Combination of MEK and AKT Inhibitors. Science signaling 6, ra68 (Aug. 2013). 
24. Nyman, E. et al. Perturbation Biology Links Temporal Protein Changes to Drug Responses in a Melanoma Cell Line. en. PLOS Computational Biology 16, e1007909 (July 2020).

25. Saez-Rodriguez, J. et al. Comparing Signaling Networks between Normal and Transformed Hepatocytes Using Discrete Logical Models. Cancer research 71, 5400-5411 (Aug. 2011).

26. Korkut, A. et al. Perturbation Biology Nominates Upstream-Downstream Drug Combinations in RAF Inhibitor Resistant Melanoma Cells. eLife 4, 1-31 (2015).

27. Soule, H. D. et al. Isolation and Characterization of a Spontaneously Immortalized Human Breast Epithelial Cell Line, MCF-10. Cancer Research 50, 6075-86 (1990).

28. Di Nicolantonio, F. et al. Replacement of Normal with Mutant Alleles in the Genome of Normal Human Cells Unveils Mutation-Specific Drug Responses. Proceedings of the National Academy of Sciences of the United States of America 105, 20864-20869 (Dec. 2008).

29. Gustin, J. P. et al. Knockin of Mutant PIK3CA Activates Multiple Oncogenic Pathways. Proceedings of the National Academy of Sciences of the United States of America 106, 2835-2840 (Feb. 2009).

30. Haagensen, E. J. et al. Pre-Clinical Use of Isogenic Cell Lines and Tumours in Vitro and in Vivo for Predictive Biomarker Discovery; Impact of KRAS and PI3KCA Mutation Status on MEK Inhibitor Activity Is Model Dependent. en. European Journal of Cancer 56, 69-76 (Mar. 2016).

31. Torrance, C. J., Agrawal, V., Vogelstein, B. \& Kinzler, K. W. Use of Isogenic Human Cancer Cells for High-Throughput Screening and Drug Discovery. en. Nature Biotechnology 19, 940-945 (Oct. 2001).

32. Martin, T. D. et al. A Role for Mitochondrial Translation in Promotion of Viability in K-Ras Mutant Cells. en. Cell Reports 20, 427-438 (July 2017).

33. De Lint, K. et al. Sensitizing Triple-Negative Breast Cancer to PI3K Inhibition by Cotargeting IGF1R. en. Molecular Cancer Therapeutics 15, 1545-1556 (July 2016).

34. Iorio, F. et al. A Landscape of Pharmacogenomic Interactions in Cancer. Cell 166, 740-754 (2016).

35. Vis, D. J. et al. Multilevel Models Improve Precision and Speed of IC50 Estimates. Pharmacogenomics 17, 691-700 (May 2016).

36. Kholodenko, B. N. et al. Untangling the Wires: A Strategy to Trace Functional Interactions in Signaling and Gene Networks. Proceedings of the National Academy of Sciences of the United States of America 99, 12841-6 (Oct. 2002).

37. Kuhn, M. \& Wickham, H. Tidymodels: a collection of packages for modeling and machine learning using tidyverse principles. (2020).

38. Virtanen, P. et al. SciPy 1.0: Fundamental Algorithms for Scientific Computing in Python. Nature Methods 17, 261-272 (2020). 


\section{Supplementary Figures}

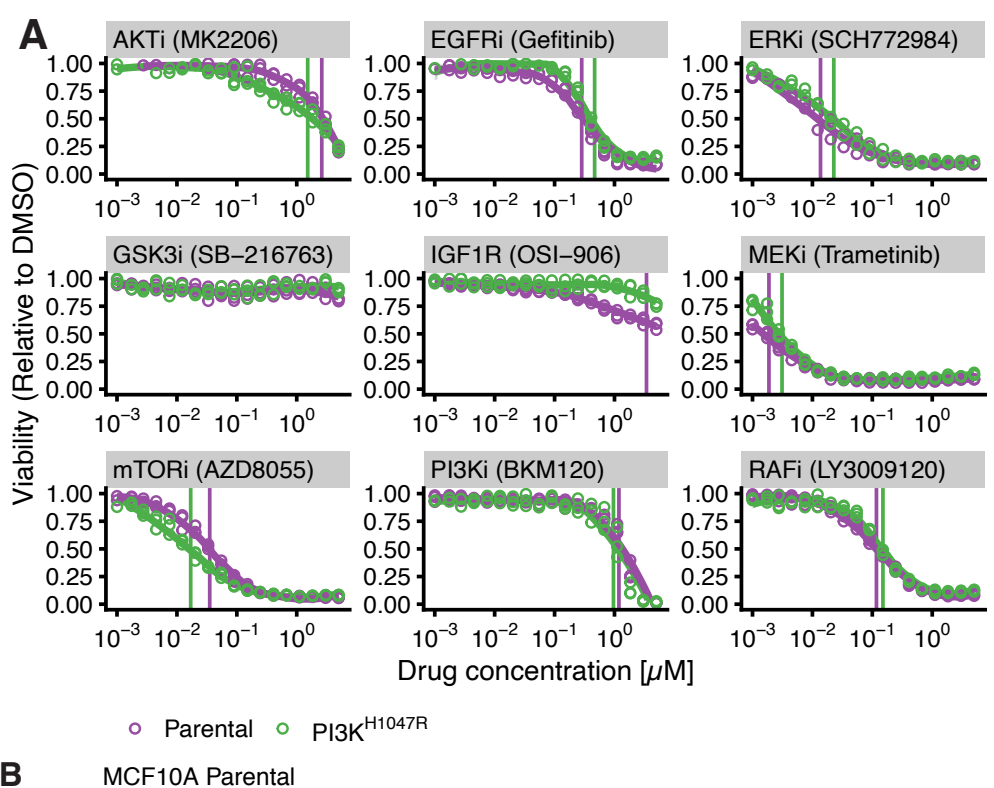

B
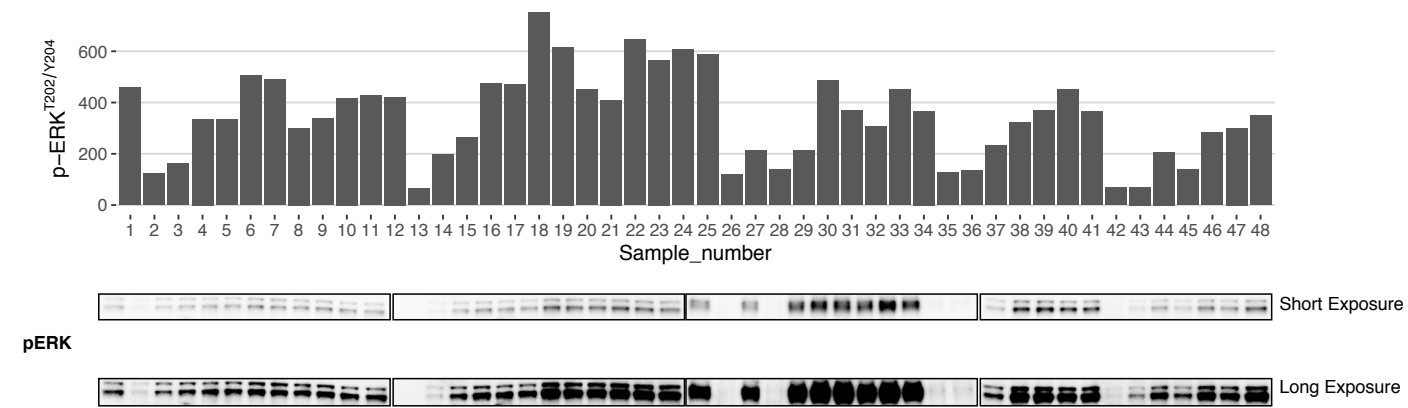

C Log2 fold change compared to DMSO
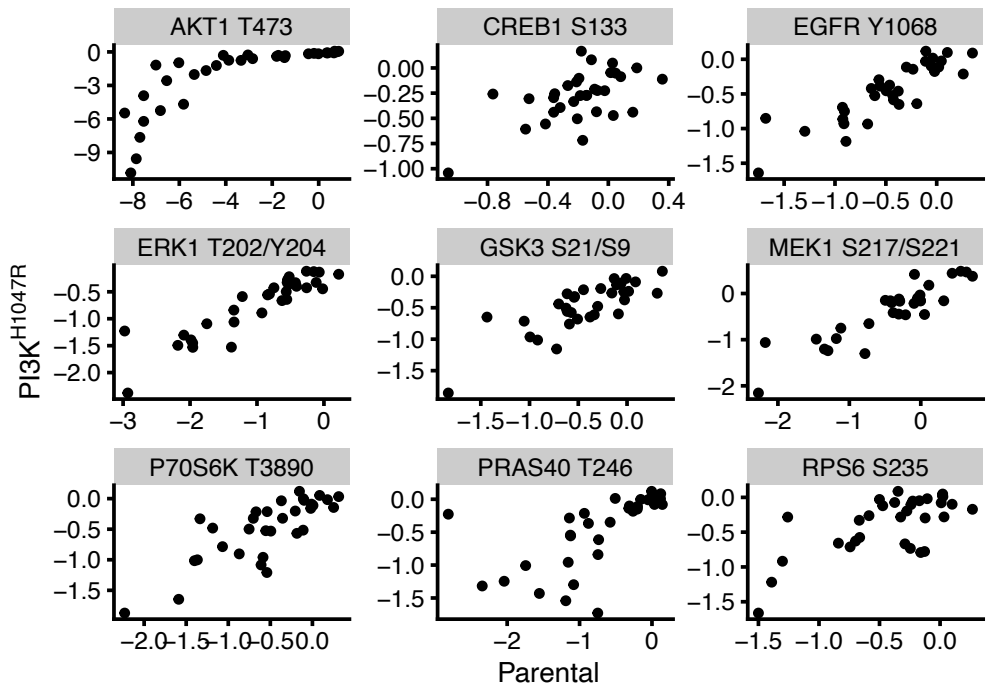

Figure S1: A. Dose-response curves of the inhibitors used in this study. B. Correlation between phosho-ERK quantification using Luminex (top) and Western blot (bottom). C. Correlation between the response in Parental (x-axis) and PI3K ${ }^{\mathrm{H} 1047 \mathrm{R}}$ (y-axis) cells. Response is defined as $\log _{2}$-fold change compared to DMSO controls. 
B

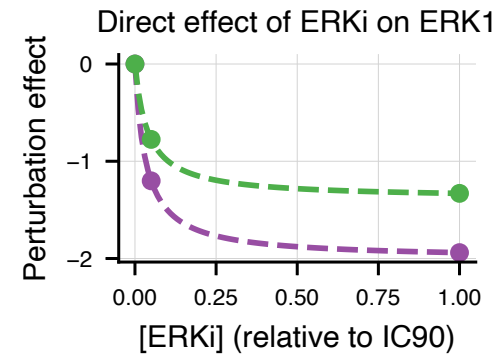

Direct effect of MEKi on MEK -

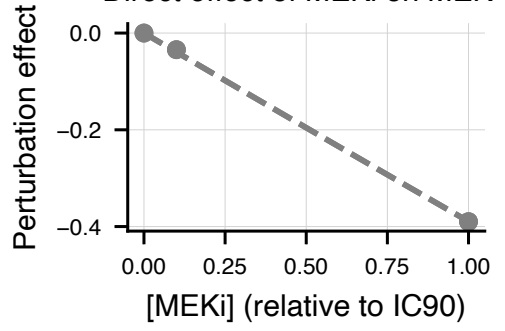

A

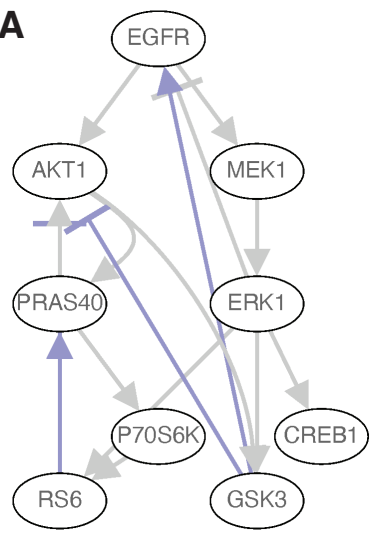

Direct effect of EGFRi on EGFI

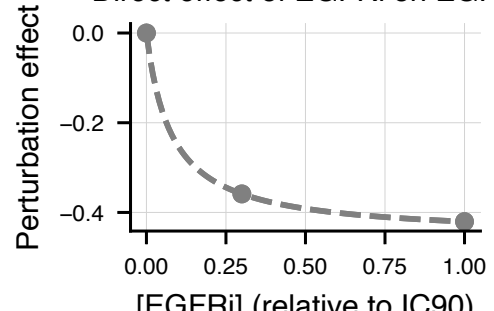

[EGFRi] (relative to IC90)

Direct effect of MEKi on ERK1

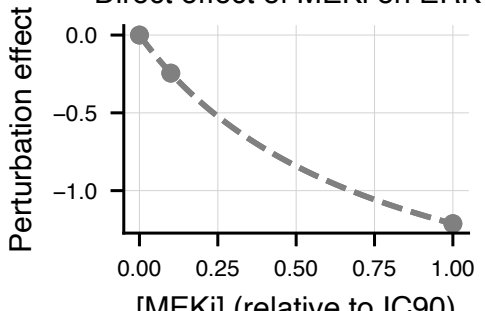

[MEKi] (relative to IC90)
Direct effect of RAFi on MEK1

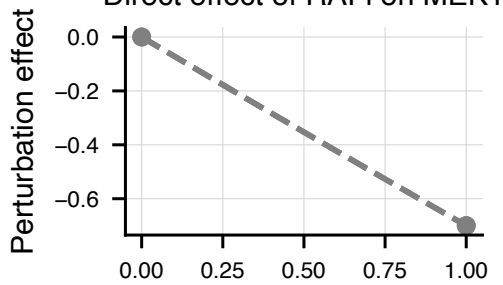

[RAFi] (relative to IC90)

Direct effect of GSK3i on GSK3

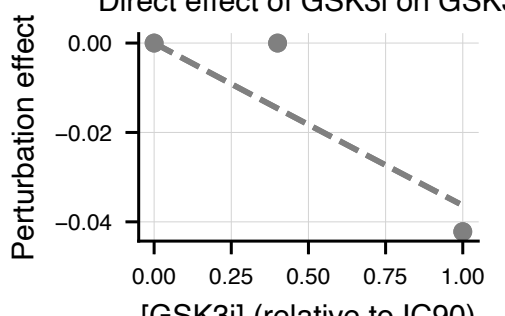

Figure S2: A. Network topology used for modeling. Edges used as prior information are indicated in gray. Edges added in a leave one out cross validation loop are indicated in purple. B. The estimated direct effect of different inhibitors on their target, as a function of applied inhibitor concentration. Points indicate the estimated effects obtained from the CNR reconstruction, at the concentrations used in the perturbation experiments. The dashed lines indicate the interpolated curves between these points. (c.f. Materials and Methods, equation (4)) 
bioRxiv preprint doi: https://doi org/10.1101/2020.1217.423240; this version posted December 18,2020 . The copyright holder for this preprint (which was not certified by peer review) is the author/funder, who has granted bioRxiv a license to display the preprint in perpetuity. It is made available under aCC-BY-NC 4.0 International license.

A Correlation between signaling responses and viability
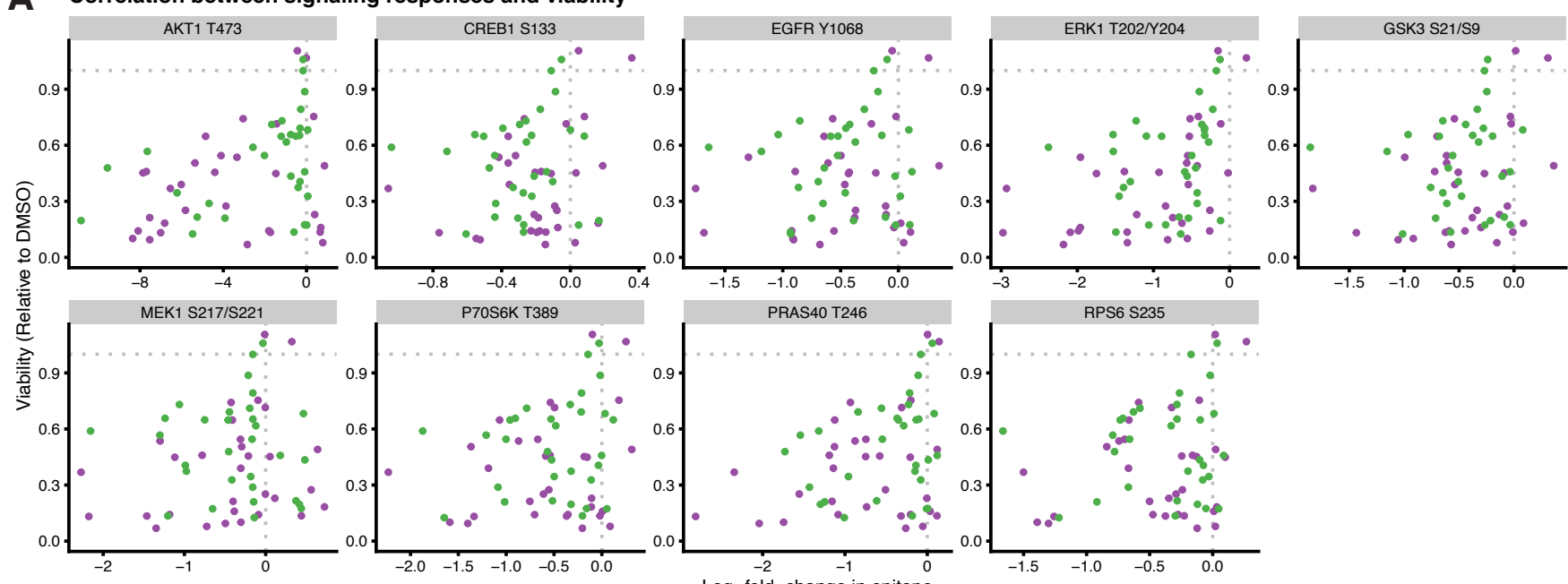

- Parental - $\mathrm{PI} 3 \mathrm{~K}^{\mathrm{H} 1047 \mathrm{R}}$

B Parameter estimates and $95 \%$ confidence intervals

Obtained using 1000 bootstraps

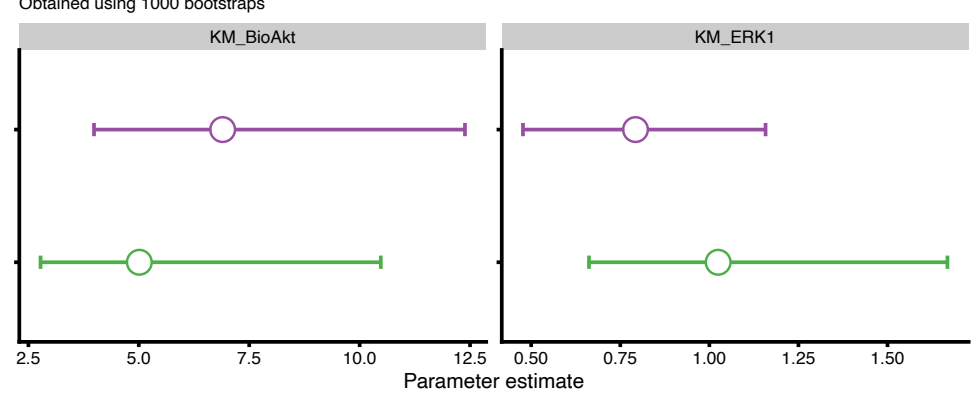

Parental $\bigcirc$ Pl3K $\mathrm{H}^{\mathrm{H} 047 \mathrm{R}}$

Figure S3: A. Correlation between node response and cell viability of all measured nodes. B. Bootstrapping intervals of the estimated values for the parameters $\mathrm{K}_{\mathrm{M}, \mathrm{AKT}}$ and $\mathrm{K}_{\mathrm{M}, \mathrm{ERK}}$ in equation (3)

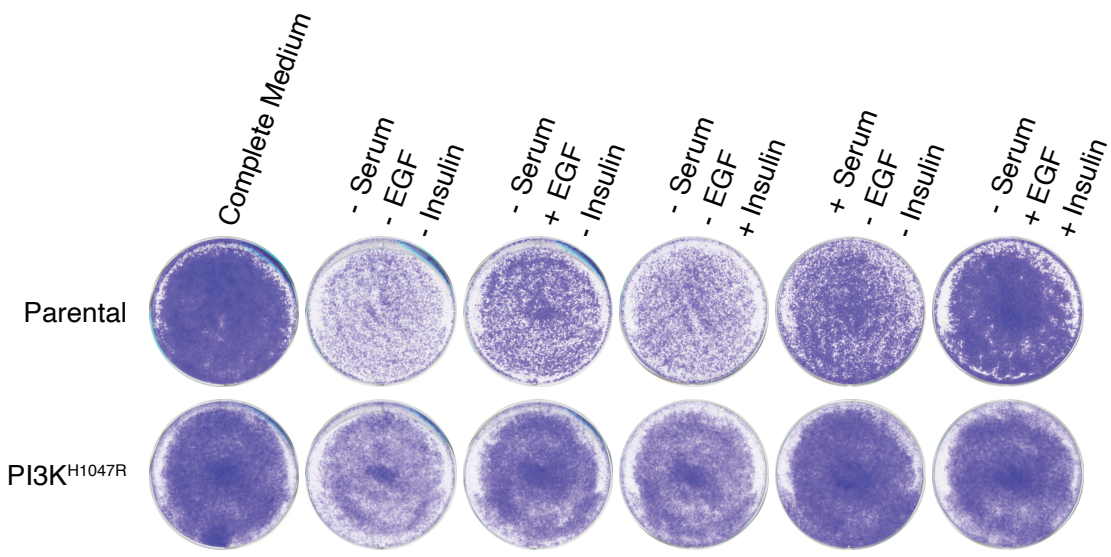

Figure S4: Growth of MCF10A parental and PI3K ${ }^{\mathrm{H} 1047 \mathrm{R}}$ cells in different growth media. In contrast to the parental cells the PI3K mutant cells grow well in the absence of serum if either Insulin or EGF is provided. 
bioRxiv preprint doi: https://doi.org/10.1101/2020.12.17.423240; this version posted December 18, 2020. The copyright holder for this preprint (which was not certified by peer review) is the author/funder, who has granted bioRxiv a license to display the preprint in perpetuity. It is made available under aCC-BY-NC 4.0 International license.

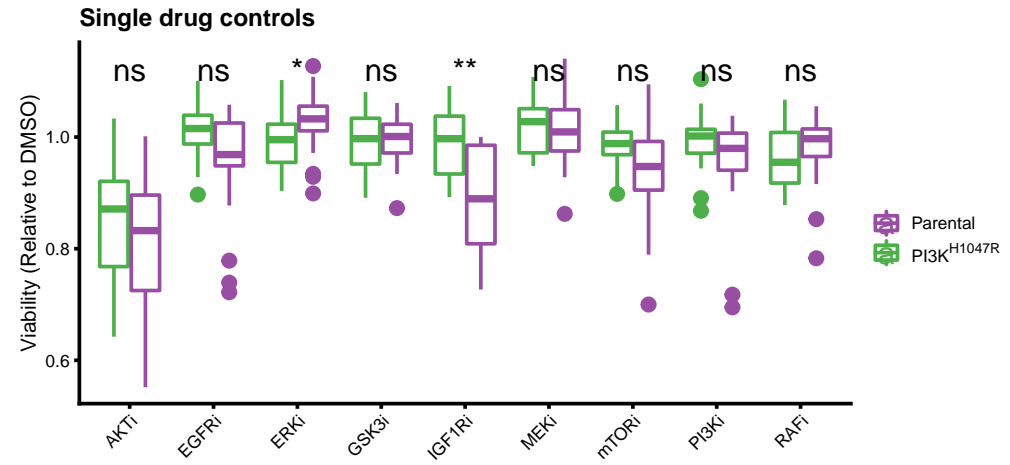

Figure S5: Viability of the low-dose single-drug controls, all measured at their $I C_{10}$. Except for IGF1Ri, none of the drugs show selectivity towards the parental cells. Treatments were performed in 8 replicates. 